\title{
Predictors of academic performance of medical undergraduate students of microbiology class in Kolkata
}

\section{Subhra Shankha Roy, Jayashree Chadalawada ${ }^{1}$ \\ Department of Microbiology, R. G. Kar Medical College and Hospital, Kolkata, West Bengal, India, ${ }^{1}$ Department of Civil Engineering, National University of Singapore, Singapore}

Address for the Correspondence:

Dr. Subhra Shankha Roy, Department of Microbiology, R. G. Kar Medical College and Hospital,

1 Kshudiram Bose Sarani, Kolkata - 700004 , West Bengal, India.

E-mail: s.shankha.roy@gmail.com

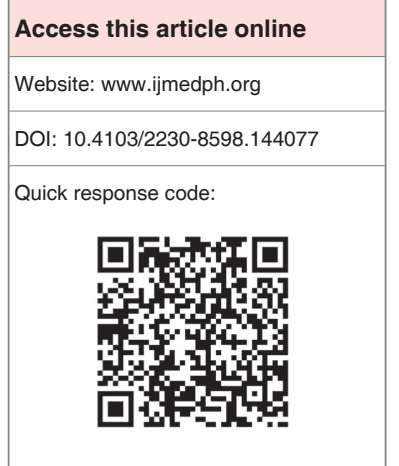

Context: Identification of the effect of valid factors on students' academic performance is of great importance to student counseling and policy making. Aims: This study was carried out to find the predictors of academic performance of $2^{\text {nd }}$ year undergraduate medical students of a renowned Medical College of Kolkata. Materials and Methods: This cross sectional study was carried out in a tertiary care teaching hospital of Kolkata. The information on factors like attendance percentage, sex, place of residence, previous academic performance of the entire batch of $2^{\text {nd }}$ year students was collected from the departments' academic records and through personal interview. The association of the above mentioned factors with students' academic performance was determined through statistical analysis using $t$-test and multiple linear regression modeling and the results were reported. Results: Academic performance is found to be weakly correlated with attendance. Better academic grade was observed for the group with high attendance percentage compared to the other with low attendance percentage $(P<0.01)$. Higher percentage of marks was observed to be scored by female students $(P<0.01)$, local students $(P<0.01)$ and high performers who were capable of successfully clearing their $1^{\text {st }}$ year's coursework in their first attempt $(P<0.01)$. Conclusion: All the factors studied in this paper which includes attendance, sex, place of residence and previous academic performance serve as predictors in understanding students' performance. Among the above mentioned, the attendance of the students is an important factor that has to be monitored and regulated through corrective actions to improve the performance of the class.

Key words: Academic performance predictors, attendance, gender, past performance, residence, undergraduate medicine

\section{INTRODUCTION}

Students pursuing medicine often face a number of social, academic, and emotional stresses, during the course of their study. Although most students successfully deal with these challenges, some are less able to survive transitions resulting in a small proportion failing to graduate at all.

One of the recent studies show that poor performers in the early years of medical school become unsuccessful doctors ${ }^{[1]}$ and are also at an increased risk for subsequent professional misconduct. ${ }^{[2]}$ In order to train a medico, the government has to invest a substantial amount of money. ${ }^{[3]}$ Therefore, for the benefit of students and society in the long run, it is important to identify the predictors of academic performance and render timely academic assistance to the students to cope up with their study.

It is a natural tendency to assume that missing classes frequently results in poor academic performances. The literature review in this area suggests that students with higher attendance perform better than students who miss class regularly. ${ }^{[4]}$ However, more recent studies suggest that a student's academic performance don't entirely depend on attendance but also on several other influencing factors. ${ }^{[5-7]}$ While the effect of absenteeism, gender and previous academic performance on students' academic grade is well-established among students of other professional courses and foreign medical students, ${ }^{[6-9]}$ the effect of the same on the grades of India medicos received little attention in the literature. Furthermore, the influence of place of residence on a student's academic performance was not discussed in detail in 
the earlier studies. In this context, a cross-sectional study is carried out to find the predictors of academic grades of medical students that involve observation of the characteristics of sophomores of a renowned Medical College of Kolkata.

\section{MATERIALS AND METHODS}

A descriptive cross-sectional study was conducted during the academic year 2012-2013 at a renowned Medical College of Kolkata after approval from the Institutional Ethical Clearance Committee. The observed population consists of 149 male and female students of $2^{\text {nd }}$ year medical undergraduate class. Of 149, two students who didn't appear for the exams were excluded. Academic performance was measured by the total percentage of marks obtained in the first and second internal examinations (theory and practical) in Microbiology. This information was collected from department academic records. Information relating to attendance was collected from department attendance register. Record of information on students' place of residence (local aid/hostel aid) was created through personal interview after obtaining informed consent. Previous academic performance data of the students' was also gathered from department records. The previous academic performance served as the indicator of students' capability to clear all the subjects of $1^{\text {st }}$ year's coursework in the first attempt.

Data entry and statistical analysis were carried out using STATA Version 12 statistical software (StataCorp 4905 Lakeway Drive, College Station, Texas 77845 USA). Distribution of data (percentage of marks obtained, attendance percentage, place of residence and past performance) was determined by plotting histograms and calculating skewness, kurtosis. As the data followed a normal distribution, unpaired $t$-test was used to analyse continuous variables and Pearson correlation coefficient was calculated to find associations. Before performing $t$-test, $F$-test was carried out to compare the variance within each group. If the variance was found to be equal a homoscedastic $t$-test was performed else heteroscedastic $t$-test was done. Two-tailed $P<0.05$ is to be considered significant. Regression analysis was performed to determine the relative contributions of attendance status, attendance in the month previous to examinations, sex, place of residence and previous academic performance to their current performance in class.

Univariate analysis was conducted to determine individual contribution of each of the above mentioned variables. Following that, a full model multiple linear regression analysis was performed to determine the association of each variable with all of the remaining variables in the model. Finally, Stepwise regression was carried out to select significant predictors of academic performance.

\section{RESULTS}

The correlation coefficient between the percentage of marks secured in examinations against attendance was weak $(r=0.27)$, but statistically significant $(P<0.005)$. Students often skip classes in preexamination period to utilize this time for more intensive study. Therefore we also calculated the pairwise correlation coefficient between the percentage of marks against attendance in the month previous to examination. This association $(r=0.22)$, though statistically significant $(P<0.05)$, was weaker than the previous case.

According to the rules and regulations of West Bengal University of Health sciences, an undergraduate student must make at least $75 \%$ attendance to be eligible to sit for professional examinations. Accordingly, total population considered for the study was divided into two groups- a group with high attendance percentage say, $75 \%$ or more $(n=75)$ and the other one with attendance percentage $<75 \%(n=72)$. A homoscedastic $t$-test showed that the group with higher attendance performed better compared with group with lower attendance $(P<0.01)$ [Table 1].

In order to investigate differences in performance based on sex and in turn, its relation to corresponding differences in attendance, the students were divided into male $(n=103)$ and female $(n=44)$ subgroups. The average examination performance of the female group was significantly better compared with the male students $(P<0.01)$. In the subgroup analysis, it was found that both in the group with high attendance percentage $(P<0.05)$ and one with low attendance percentage $(P<0.01)$, female students performed better than their male colleagues.

To find out the influence of place of residence on academic performance, the students were divided into local aid $(n=50)$ and hostel aid $(n=97)$ subgroup. A homoscedastic $t$-test showed that local aid students consistently performed better than those with hostel aid at a significant level $(P<0.01)$. Moreover comparing the group with high attendance record $(\mu=62.96 \%, 95 \%$ confidence interval [CI]: $60.38-65.53 \%$ vs. $\mu=56.74 \%, 95 \%$ CI: $54.32-59.15 \%$, $P<0.005)$ and the one with low attendance record $(\mu=55.63 \%, 95 \%$

\begin{tabular}{|c|c|c|c|c|c|c|}
\hline Variable & Subgroup & Sample size & Mean & $95 \%$ Confidence interval & $t$-value & $P$ value \\
\hline \multirow[t]{2}{*}{ Attendance } & $<75 \%$ & 71 & 53.01 & $50.58-55.44$ & -3.24 & $<0.01$ \\
\hline & $>75 \%$ & 76 & 58.25 & $56.13-60.38$ & & \\
\hline \multirow[t]{2}{*}{ Gender } & Female & 44 & 60.67 & $58.64-62.71$ & 4.87 & $<0.01$ \\
\hline & Male & 103 & 53.61 & $51.54-55.67$ & & \\
\hline \multirow[t]{2}{*}{ Residence } & Hostel aid & 97 & 53.35 & $51.38-55.32$ & -4.18 & $<0.01$ \\
\hline & Day scholar & 50 & 60.32 & $57.72-62.92$ & & \\
\hline \multirow[t]{2}{*}{ Previous performance } & Poor performers & 24 & 42.51 & $39.56-45.46$ & -8.57 & $<0.01$ \\
\hline & High performers & 123 & 58.30 & $56.79-59.81$ & & \\
\hline
\end{tabular}


CI: $50.35-60.90 \%$ vs. $\mu=49.75 \%, 95 \%$ CI: 46.86-52.64\%, $P<0.05)$, students with local aid outperformed their colleague with hostel aid.

The Pearson correlation coefficient for percentage of marks against attendance for local aid and hostel aid students are 0.37 and 0.21 respectively at a significant level $(P<0.05)$.

To find out the effects of previous academic performance on current performance, the students were divided into two groups-poor performers, who failed to clear all the subjects of $1^{\text {st }}$ year MBBS coursework in their first attempt and high performers, who cleared all the $1^{\text {st }}$ year subjects in their first attempt. High performers scored significantly better in the microbiology examination compared to the poor performers of the previous year $(P<0.01)$.

The univariate analysis showed statistically significant contributions of all variables when considered individually, which was followed by full-model multivariate analysis. Full-model multivariate analysis showed how attendance status, sex, place of residence, and previous performance contributes significantly to a final model. Attendance in the month before examinations also showed a significant contribution in univariate regression model but it failed to show a positive association in multilinear regression model. These variables were the ones selected for stepwise multiple regression analysis resulting in $r^{2}=0.469$ [Table 2].

\section{DISCUSSION}

Identifying the effect of various factors on students' academic performance is of great importance to educators and psychologists. This study shows that students' better performance in Microbiology exam is associated with high attendance percentage, female gender, residence (local aid) and successful performance in $1^{\text {st }}$ year. All these terms together can predict $47 \%$ of the variability of present academic performance.

Though, in univariate regression model, attendance in the month before examinations was associated positively with performance but it failed to show a positive association in multiple linear regression

\begin{tabular}{|c|c|c|c|c|}
\hline \multirow[t]{2}{*}{$\begin{array}{l}\text { Variables in } \\
\text { percentage }\end{array}$} & \multicolumn{2}{|c|}{$\begin{array}{l}\text { Univariate } \\
\text { analysis }\end{array}$} & \multicolumn{2}{|c|}{$\begin{array}{c}\text { Multivariate } \\
\text { analysis }\end{array}$} \\
\hline & B & $t$-value & $\beta$ & $t$-value \\
\hline Attendance status & 5.240 & $3.25^{\star *}$ & 2.534 & $1.98^{*}$ \\
\hline Place of residence & 6.966 & $4.18^{* *}$ & 5.607 & $4.25^{\star *}$ \\
\hline Sex & -7.066 & $4.09^{* *}$ & 4.977 & $3.55^{\star *}$ \\
\hline Previous performance & 15.787 & $8.57^{* *}$ & 13.032 & $7.54^{* *}$ \\
\hline $\begin{array}{l}\text { Attendance before } \\
\text { examination }\end{array}$ & 0.695 & $2.71^{* *}$ & 0.185 & 0.77 \\
\hline Constant & & & 45.090 & $21.90^{\star *}$ \\
\hline $\mathrm{R}^{2}$ & & & 0.47 & \\
\hline$n$ & & & 147 & \\
\hline
\end{tabular}

$* P<0.05, * * P<0.01, \beta=$ Regression coefficient model. The reason for this is the colinearity that exists between the attendance in preexamination period with the attendance status. Previous studies reported that high percentages of marks are secured by students who attend classes regularly compared to those who failed to do so. Jaykaran et al. ${ }^{[10]}$ in his study reported a moderate correlation between attendance and marks obtained in pharmacology by the Indian medical undergraduate students (Pearson correlation coefficient $=0.578, P=0.00)$. BinSaeed et al. , $^{[1]]}$ also demonstrated that students without a history of absenteeism had a higher mean grade point average (GPA) in comparison with students with a history of absenteeism. But the common limitation in the above mentioned studies is the usage of bivariate analysis.

In literature a consistent finding is that women likely to perform better than the men counterparts in their medical training. This trend also continues even in clinical assessments. In a study done by Sawair et al. ${ }^{[12]}$ in Jordan by making observations on 413 dentistry students, it was found that the female students have a significantly better GPA compared to the male students. The reason for this difference is not clear. It can be attributed to the difference in motivational, academic, and demographic factors between men and women that influence their performance.

Jaykaran et al. ${ }^{[10]}$ reported a comparable academic performance of day scholar and hostel aid students which differ from the result of this study where day scholar students performed better than their colleagues with hostel aid. The reason for this difference though not investigated in this study could be because of the rural-urban background of students and the medium of instruction at their schools. In literature, it is reported that in undergraduate/graduate studies, students from English medium schools perform better than non English medium students. ${ }^{[13]}$ Educational outcomes of urban students are also better than the rural students. ${ }^{[14]}$ As students who are day scholars are mostly from Kolkata and nearby urban areas, they are more likely to have come from English medium schools, which explain their better performance compared to students with hostel aid. The place of residence appears to be a significant predictor as this study is conducted among $2^{\text {nd }}$ year undergraduate students. There is a possibility that in the future, this difference may disappear owing to the practice of exposure to lectures delivered in English. Measures of previous academic performance can include a student's high school performance or their performance in the $1^{\text {st }}$ year of medical college. Students entering medical college tend to be at the top end of the possible range of scores in their high school. This small range of high school score affects the statistical correlation between present and past academic performances. Due to this limitation of the premedical score, it was not considered in this study rather a more reliable parameter-academic success in $1^{\text {st }}$ year was considered. Our finding that previous academic performance contributes to present performance is confirmed by other studies. ${ }^{[6,15-17]}$

Our study has limitations. As this is a pilot study we concentrated on few variables only. We didn't take account of variables such as medium of instruction in school, socio-economic status, and memory in our study. Hence, there is a possibility that these factors 
or some other uncontrolled factors may act as a confounder and have contributed to these effects. In the future, we need to perform a more comprehensive evaluation taking into account of more variables other than those considered for this study say, learning style, memory, stress coping up pattern, study pattern, personality, age, medium of instruction in school, rural/urban background, socio-economic status etc. Furthermore, increasing the population size for the study will help to predict the outcome more accurately.

Taken together, our study demonstrates that medical undergraduate students' better performance in Microbiology exam is associated with high attendance percentage, female gender, residence (local aid) and successful performance in $1^{\text {st }}$ year. Among the above mentioned, the attendance of the students is the only modifiable factor that has to be monitored and regulated through corrective actions to improve the performance of the class.

\section{REFERENCES}

1. Papadakis MA, Teherani A, Banach MA, Knettler TR, Rattner SL, Stern DT, et al. Disciplinary action by medical boards and prior behavior in medical school. N Engl J Med 2005;353:2673-82.

2. Yates J, James D. Risk factors at medical school for subsequent professional misconduct: Multicentre retrospective case-control study. BMJ 2010 27;340:c2040.

3. Maslov Kruzicevic S, Barisic KJ, Banozic A, Esteban CD, Sapunar D, Puljak L. Predictors of attrition and academic success of medical students: A 30-year retrospective study. PLoS One 2012;7:e39144.

4. Hammen CS, Kelland JL. Attendance and grades in a human physiology course. Am J Physiol 1994;267:S105-8.

5. Massingham P, Herrington T. Does attendance matter? An examination of student attitudes, participation, performance and attendance pre-amble. Practice 2006;3:82-103.

6. Frischenschlager O, Haidinger G, Mitterauer L. Factors associated with academic success at Vienna Medical School: Prospective survey. Croat Med J 2005;46:58-65.
7. Mills C, Heyworth J, Rosenwax L, Carr S, Rosenberg M. Factors associated with the academic success of $1^{\text {st }}$ year health science students. Adv Health Sci Educ Theory Pract 2009;14:205-17.

8. Hidayat L, Vansal S, Kim E, Sullivan M, Salbu R. Pharmacy student absenteeism and academic performance. Am J Pharm Educ 2012;76:8.

9. Gatherer D, Manning FC. Correlation of examination performance with lecture attendance: A comparative study of $1^{\text {st }}$-year biological sciences undergraduates. Biochem Educ 1998;26:121-3.

10. Jaykaran, Yadav P, Chavda N, Kantharia ND. Factors associated with performance of $2^{\text {nd }}$ year student in pharmacology examinations. J Pharmacol Pharmacother 2011;2:123-5.

11. BinSaeed AA, Al-Otaibi MS, Al-Ziyadi HG, Babsail AA, Shaik SA. Association between student absenteeism at a Medical college and their academic grades. Med Sci Educ 2009;19:155-9.

12. Sawair FA, Baqain ZH, Al-Omari IK, Wahab FK, Rajab LD. Effect of gender on performance of undergraduate dental students at the University of Jordan, Amman. J Dent Educ 2009;73:1313-9.

13. Chur-Hansen A, Vernon-Roberts J, Clark S. Language background, English language proficiency and medical communication skills of medical students. Med Educ 1997;31:259-63.

14. Ramos R, Duque JC, Nieto S. Decomposing the rural-urban differential in student acheivement in Colombia using PISA microdata; IZA, 2012. Available from: http://www.ftp.iza.org/dp6515.pdf [Last accessed on 2014 July 16].

15. Ferguson E, James D, Madeley L. Factors associated with success in medical school: Systematic review of the literature. BMJ 2002 20;324: 952-7.

16. Egbewale BE, Adeeyo OA, Ogunro PS, Olowu AO, Adeoti ML, Adewole TA. Predictors of students'performance in the pre-clinical MBBS programme in a Nigerian University. Niger Postgrad Med J 2009;16: 245-50.

17. Hall FR, Bailey BA. Correlating students' undergraduate science GPAs, their MCAT scores, and the academic caliber of their undergraduate colleges with their $1^{\text {st }}$-year academic performances across five classes at Dartmouth Medical School. Acad Med 1992;67:121-3.

How to cite this article: Roy SS, Chadalawada J. Predictors of academic performance of medical undergraduate students of microbiology class in Kolkata. Int J Med Public Health 2014;4:392-5.

Source of Support: Nil, Conflict of Interest: None declared. 